\title{
Interval estimation of reliability parameters in reliability engineering
}

\author{
Ming Han \\ School of Sciences, Ningbo University of Technology, Zhejiang, Ningbo 315211, China \\ E-mai:hanming618@21cn.com
}

Keywords: reliability engineering, failure rate, exponential distribution, zero-failure data, two-sided confidence limits.

\begin{abstract}
For the reliability evaluation of such expensive and high quality products as those used in aviation and spacecraft, this study gives the two-sided confidence limits estimation of reliability parameters for exponential distribution: average life, reliability and reliability life, in the case of zero-failure data. Calculation performed on practical problems show that the provided method is feasible and easy to operation in reliability engineering.
\end{abstract}

\section{Introduction}

In the area related to reliability of industrial products, for the life testing of products, engineers often deal with censored data, which sometimes have small sample size or have been censored. Also, the products of interest have high reliability. Since Lindley and Smith (1972) introduced the idea of hierarchical prior distribution, some results have been obtained on hierarchical Bayesian method to deal with lifetime data. But all those results obtained by means of hierarchical Bayesian methods involve complicated integration compute. Though some computing methods such as Markov Chain Monte Carlo (MCMC) are available, doing integration is still very inconvenient for practical problems.

Han (1997) developed some methods to construct hierarchical prior distribution. Recently, hierarchical Bayesian methods have been applied to data analysis. For more details, see Ando and Zellner (2010), Richard (2011). Especially some other results on hierarchical Bayesian methods have been proposed to deal with zero-failure data (Han and Li, 1999), something that has long been recognized since the article of Martz and Waller (1979) appeared. For a detailed review of zero-failure data problem, refer to Han (2002).

Consider type I censored life testing $m$ time, denote the censored times as $t_{i}(i=1,2, \cdots, m)$, the corresponding sample numbers as $n_{i}$, if no failure in these samples are observed in the testing process, then $\left\{\left(n_{i}, t_{i}\right), i=1,2, \cdots, m\right\}$ is called the zero-failure data.

In the case of grouped data, the two-sided confidence limit of estimation of the failure rate is described in Section 2. In the case of not grouped data, the two-sided confidence limit of estimation of the failure rate is described in Section 3. In Section 4, is two applications example is given. Section 5 is the conclusion.

\section{In the case of grouped data}

Let us suppose the life of a product follows an exponential distribution with probability density function

$$
f(t)=\lambda \exp (-t \lambda) t>0
$$

where $\lambda>0$ is the failure rate of the exponential distribution.

Theorem 1. For the testing the zero-failure data set $\left\{\left(n_{i}, t_{i}\right), i=1,2, \cdots, m\right\}$ from $m$ times type I censored testing of life distribution (1). In the of grouped data, if the credible level is $0<\alpha<1$, then we have the following two conclusions:

(1) two-sided confidence lower limit and confidence upper limit of average life $\theta$, respectively, 


$$
\theta_{\mathrm{L}}=\frac{\sum_{i=1}^{m} n_{i} t_{i}}{-\ln \frac{\alpha}{2}}, \quad \theta_{\mathrm{U}}=\frac{\sum_{i=1}^{m} n_{i} t_{i}}{-\ln \left(1-\frac{\alpha}{2}\right)}
$$

(2) two-sided confidence lower limit and confidence upper limit of reliability R , respectively,

$$
\mathrm{R}_{\mathrm{L}}(t)=\exp \left\{\frac{t \ln \frac{\alpha}{2}}{\sum_{i=1}^{m} n_{i} t_{i}}\right\}, \quad \mathrm{R}_{\mathrm{U}}(t)=\exp \left\{\frac{t \ln \left(1-\frac{\alpha}{2}\right)}{\sum_{i=1}^{m} n_{i} t_{i}}\right\} ;
$$

(3) two-sided confidence lower limit and confidence upper limit of reliability life $t(R)$, respectively,

$$
t_{L}(R)=\frac{\ln R}{\ln \frac{\alpha}{2}} \sum_{i=1}^{m} n_{i} t_{i}, \quad t_{U}(R)=\frac{\ln R}{\ln \left(1-\frac{\alpha}{2}\right)} \sum_{i=1}^{m} n_{i} t_{i} .
$$

\section{In the case of not grouped data}

Theorem 2. For the testing the zero-failure data set $\left\{\left(n_{i}, t_{i}\right), i=1,2, \cdots, m\right\}$ from $m$ times type I censored testing of life distribution (1). In the case of not grouped data, if the credible level is $0<\alpha<1$, then we have the following two conclusions:

(1) two-sided confidence lower limit and confidence upper limit of average life $\theta$, respectively,

$$
\theta_{\mathrm{L}}=\frac{\sum_{i=1}^{m} t_{i}}{-\ln \frac{\alpha}{2}}, \quad \theta_{\mathrm{U}}=\frac{\sum_{i=1}^{m} t_{i}}{-\ln \left(1-\frac{\alpha}{2}\right)} ;
$$

(2) two-sided confidence lower limit and confidence upper limit of reliability R , respectively,

$$
\mathrm{R}_{\mathrm{L}}(t)=\exp \left\{\frac{t \ln \frac{\alpha}{2}}{\sum_{i=1}^{m} t_{i}}\right\}, \quad \mathrm{R}_{\mathrm{U}}(t)=\exp \left\{\frac{t \ln \left(1-\frac{\alpha}{2}\right)}{\sum_{i=1}^{m} t_{i}}\right\} ;
$$

(3) two-sided confidence lower limit and confidence upper limit of reliability life $t(R)$, respectively,

$$
t_{L}(R)=\frac{\ln R}{\ln \frac{\alpha}{2}} \sum_{i=1}^{m} t_{i}, \quad t_{U}(R)=\frac{\ln R}{\ln \left(1-\frac{\alpha}{2}\right)} \sum_{i=1}^{m} t_{i} .
$$

\section{Application Example}

The following two application examples are given.

Application Example 1. Consider the testing data from electronic products in type I censored testing, results all samples without a failure, zero-failure data are shown in Table1 (time unit: hour), the life of such type of electronic products has an exponential distribution.

Table 1 Zero-failure data of electronic products

\begin{tabular}{ccccccc}
\hline$i$ & 1 & 2 & 3 & 4 & 5 & 6 \\
\hline$t_{i}$ & 450 & 650 & 850 & 1050 & 1250 & 1450 \\
$n_{i}$ & 2 & 2 & 2 & 3 & 3 & 3 \\
\hline
\end{tabular}


(1)When confidence level, respectively $0.60,0.70,0.80,0.90$, interval estimation of average life $\theta$ are given;

(2) When confidence level, respectively $0.60,0.70,0.80,0.90, \theta=500(h)$, interval estimation of reliability $\mathrm{R}$ are given;

(3) When confidence level, respectively $0.60,0.70,0.80,0.90, \mathrm{R}=0.8$, interval estimation of reliability life $t(R)$ are given.

According to Theorem 1, and Table 1, we can obtain: interval estimation of average life $\theta$, interval estimation of reliability $\mathrm{R}$ and interval estimation of reliability life $t(R)$. Some numerical results of are listed in Table 2.

Table 2 Results of interval estimation of reliability parameters

\begin{tabular}{ccccc}
\hline $1-\alpha$ & 0.6 & 0.7 & 0.8 & 0.9 \\
\hline$\left(\theta_{L}, \theta_{U}\right)$ & $(9413.2,16534.0$ & $(7985.8,17705.5$ & $(6579.5,18972.8$ & $(5057.1,20350.8)$ \\
$\left(R_{L}(500), R_{U}(500)\right)$ & $(0.9482,0.9927)$ & $(0.9393,0.9946)$ & $(0.9268,0.9965)$ & $(0.9059,0.9983)$ \\
$\left(t_{L}(0.8), t_{U}(0.8)\right)$ & $(2100.5,3689.4)$ & $(1781.9,3950.8)$ & $(1468.2,4233.6)$ & $(1128.5,4541.1)$ \\
\hline
\end{tabular}

Application Example 2. Consider the testing data from electronic products in type I censored testing, results all samples without a failure, zero-failure data are shown inTable3 (time unit: hour), the life of such type of electronic products has an exponential distribution.

Table 3 Zero-failure data of electronic products

\begin{tabular}{ccccccccccc}
\hline$i$ & 1 & 2 & 3 & 4 & 5 & 6 & 7 & 8 & 9 & 10 \\
\hline$t_{i}$ & 200 & 250 & 300 & 350 & 400 & 450 & 500 & 550 & 600 & 650 \\
\hline
\end{tabular}

(1)When confidence level, respectively $0.60,0.70,0.80,0.90$, interval estimation of average life $\theta$ are given;

(2) When confidence level, respectively $0.60,0.70,0.80,0.90, \theta=300(h)$, interval estimation of reliability $\mathrm{R}$ are given;

(3) When confidence level, respectively $0.60,0.70,0.80,0.90, \mathrm{R}=0.8$, interval estimation of reliability life $t(R)$ are given.

According to Theorem 2, and Table 3, we can obtain: interval estimation of average life $\theta$, interval estimation of reliability $\mathrm{R}$ and interval estimation of reliability life $t(R)$. Some numerical results of are listed in Table 4.

Table 4 Results of interval estimation of reliability parameters

\begin{tabular}{ccccc}
\hline $1-\alpha$ & 0.6 & 0.7 & 0.8 & 0.9 \\
\hline$\left(\theta_{L}, \theta_{U}\right)$ & $(2640.7,4638.2)$ & $(2240.2,4966.8)$ & $(1845.7,5322.4)$ & $(1418.7,5708.9)$ \\
$\left(R_{L}(300), R_{U}(300)\right)$ & $(0.8926,0.9843)$ & $(0.8746,0.9886)$ & $(0.8500,0.9926)$ & $(0.8094,0.9964)$ \\
$\left(t_{L}(0.8), t_{U}(0.8)\right)$ & $(589.2,1034.9)$ & $(499.9,1108.3)$ & $(411.8,1187.6)$ & $(316.5,1273.9)$ \\
\hline
\end{tabular}

\section{Conclusion}

This paper, in the case of grouped data and not grouped data, are given respectively the two-sided confidence limits estimation of reliability parameters for exponential distribution: average life, reliability and reliability life, in the case of zero-failure data. According to Theorem 1 and Theorem 2, in the case of zero-failure data, we can obtain the interval estimation of reliability parameters for exponential distribution.

Reviewing the application example, it is show that the proposed method is feasible and convenient in engineering application. 


\section{Acknowledgments}

This work was supported partly by the Ningbo Natural Science Foundation of China (No.2013A610108).

\section{References}

[1]T. Ando, A. Zellner. .Hierarchical Bayesian Analysis of the Seemingly Unrelated Regression and Simultaneous Equations Models Using a Combination of Direct Monte Carlo and Importance Sampling Techniques. Bayesian Analysis. Vol.5 (2010), 65-96.

[2]M. Han. The structure of hierarchical prior distribution and its applications. Chinese Operations Research and Management Science. Vol.6 (1997), 31-40.

[3]M. Han, Y. Q. Li. Hierarchical Bayesian estimation of the products reliability based on zero-failure data. Journal of Systems Science and Systems Engineering. Vol.8 (1999), 467-471.

[4]M. Han. The advances of relaibility for zero-failure data. Chinese Advances in Mathematics. Vol.31 (2002), 7-19.

[5]D. V. Lindley, A. F. M. Smith. Bayes estimates for the linear model. Journal of the Royal Statistical Society. Series B. Vol.34 (1972),1-41.

[6]H. F. Martz, R.A.Waller. A Bayes zero-failure (BAZE) reliability demonstration testing procedure. Journal of Quality Technology. Vol.11 (1979), 128-137.

[7] D. M. Richard. A Bayesian hierarchical model for the measurement of working memory capacity. Journal of Mathematical Psychology. Vol.55 (2011), 8-24. 